\title{
Fault diagnosis in gear using wavelet envelope power spectrum
}

\author{
M. Lokesha ${ }^{1 *}$, Manik Chandra Majumder ${ }^{2}$, K. P. Ramachandran ${ }^{3}$, Khalid Fathi Abdul Raheem ${ }^{4}$ \\ ${ }^{1,3,4}$ Department of Mechanical Engineering, Caledonian College of Engineering, OMAN, \\ ${ }^{2}$ Department of Mechanical Engineering, National Institute of Technology, Durgapur, INDIA, \\ Corresponding author: lokjay251@yahoo.com,Tel: +968-92368754
}

\begin{abstract}
In recent years, improvement has been achieved in vibration signal processing, using wavelet analysis for condition monitoring and fault diagnosis. The use of wavelet analysis has proven to be efficient to detect faults in vibration signals with nonstationary, transient characteristics/ components. An experimental data set is used to compare the diagnostic capability of the fast Fourier transform power spectrum to the wavelet envelope power spectrum as respectively computed using Laplace and Morlet wavelet functions. The gear testing apparatus was used for experimental studies to obtain the vibration signal from a healthy gear and a faulty gear.
\end{abstract}

Keywords: Fast Fourier transform, Continuous wavelet transform, Envelope power spectrum, Wavelet.

DOI: http://dx.doi.org/10.4314/ijest.v3i8.13

\section{Introduction}

In modern industries, the reduction in productivity and increase in maintenance cost are mainly due to fault in the machineries. The predictive maintenance techniques are most widely used to reduce the maintenance cost and increase the production. As machineries in modern industries are expected to run continuously, unexpected down time of rotating machinery reduces production. Since most of the machineries contain gear box assembly, it is imperative to develop suitable condition monitoring technique to prevent malfunctioning and breakage during operation. Early detection of fault is very important to avoid malfunction of machine that would leads to serious damage to the complete system. Fault diagnosis is an important process in condition monitoring of gear box which avoids serious damage. Vibration based fault detection and diagnosis analysis is a broadly used condition monitoring technique for gears as well as other mechanical components like bearings (Omar and Gaouda, 2009; AbuMahfouz, 2007). To analyze vibration signals different techniques such as time, frequency and time-frequency domain techniques are extensively used (Wandel, 2006; Choy et al., 1994; Saravanan and Ramachandran, 2010). Time domain techniques concentrates on statistical characteristics like kurtosis, peak level, crest factor standard deviation, impulse factor, shape factor etc. While the frequency domain uses fast Fourier transform (FFT) of time domain signal, power spectrum, cepstrum etc to assess the condition based on the frequency content of the signal.

These techniques are based on the assumptions of stationary vibration signals. Fourier transform (FT) represents a signal by a family of complex exponents with infinite time duration therefore FT is useful in identifying harmonic signals, however due to its constant time and frequency resolution, it is weak in analyzing transient signal components.(Tse et al., 2004). Presence of a fault in a gear tooth produces impacts, which may result in non-stationary signal due to transient modification of the vibration signal (Dalpiaz et al., 1998; Randall, 1982). Usually, these non-stationary components contain abundant information about machine faults; therefore, it is important to analyze the non-stationary signals. The non-stationary nature of the signal suggests the use of time-frequency techniques, which make it possible to look at the time evolution of the signal's frequency content (Dalpiaz et al., 1998; Saravanan et al., 2010). FFT based condition monitoring techniques are not suitable for non-stationary signal analysis (Pan and Sas, 1996). 
The analysis of the non-stationary signals developed by the complex machines needs new techniques which go beyond classical Fourier approach. There exist a lot of different time variant methods; some are reviewed in Wandel (2006), Peng and Chu (2004) and Omar and Gaouda (2009).

A time-frequency distribution describes the energy distribution of a signal in both the time and the frequency domain. The Spectrogram is an energy density spectrum produced by the short-time Fourier transform (STFT), which is simply the FT applied to many short time windows. Narrow time windows used to find fast variations results in poor frequency resolution and the tradeoff between time and frequency resolution is the main disadvantage of the STFT. It is particularly difficult to analyze signals with both low and high frequency components (Wandel, 2006). In comparison with STFT, the Wigner-Ville distribution (WVD) provides better resolution in the time-frequency plane, but at the cost of severe interference terms. The cross term interference is the main deficiency of the WVD. (Wandel, 2006; Peng et al., 2004).

The wavelet transform (WT) provides powerful multi-resolution analysis in both time and frequency domains and thereby becomes a favored tool to extract the transient signal components/ characteristics features of non-stationary vibrations signals produced by the faults. Wavelet analysis gives better information to fault compared with Fast Fourier Transform (FFT) spectrum (Peng et al., 2003, Purushotham et al., 2005, Shi et al., 2004). Scaled and shifted wavelet window produces better detection capability over fixed size window of STFT. In WT the non-stationary vibration signal to be analyzed is filtered into different frequency bands, which are split into segments in time domain and their frequency contents and energy are analyzed. During the analysis the wavelet is transformed in time to select the part of the signal to be analyzed, then dilated/ expanded or contracted/compressed in order to focus on a given range or number of oscillations. When the wavelet is expanded, it focuses on the signal components with low frequencies and when compressed on the components with high frequencies. Due to the compression and expansion of the wavelet, the WT performs a time scale decomposition of the signal into weighted set of scaled wavelet functions.

The Wavelet analysis results in a series of wavelet coefficients, which indicate the correlation of the signal with the particular wavelet. In order to extract the fault features of the signal more effective appropriate wavelet base function should be selected (Nikolaou and Antoniadis, 2002; Junsheng et al., 2005). The main investigation areas related to use of wavelet analysis in the fault detection and diagnosis process include the selection of the most appropriate wavelet base function with the more similarity to the fault feature to be identified and how to analyze the generated wavelet coefficients to extract the fault related features. A number of wavelet based functions are proposed for mechanical fault detection with high sensitivity. Morlet wavelet and Impulse wavelet are applied to extract the gear and rolling element ball bearing fault features and these wavelet parameters are optimized based on maximum kurtosis to enhance the fault detection process.(Lin et al., 2003; Al-Raheem et al., 2006; Khalid et al., 2009; Khalid, 2007).

Laplace wavelet is a complex, single sided damped exponential formulated as an impulse response of a single mode system to be similar to data features commonly encountered in health monitoring tasks. It has been applied to the vibration analysis of an aircraft for aerodynamic and structural testing (Freudinger et al., 1998) and to diagnose the wear of the intake valve of an internal combustion engine (Yanyang et al., 2005).

Vibration monitoring is used in diverse fault monitoring schemes. Still, in practice, comprehensive use of on-line condition monitoring systems does not exist. The Haar wavelet and Morlet wavelet are compared for signal processing as the Haar wavelet is easy to implement, but it does not provide smoothing and the Morlet wavelet provides smoothing, but it is not a very flexible tool in signal analysis. (Smith et al., 2006)

It is well known that the most important components in gear vibration spectra are the tooth meshing frequency and its harmonics, together with sidebands due to modulation phenomena. The increment in the number and amplitude of such sidebands may indicate a fault condition. It may serve as a tool for aiding the gear fault diagnosis (Randall, 1982; van Khang, 2004; Prokop and Mohr, 2003).

This paper proposes that wavelet envelope power spectra may be used to detect localised gear tooth defects. While the wavelet envelope power spectrum has been investigated in the context of detecting bearing faults, its application to gear fault monitoring is novel. It is also investigated how the wavelet parameters can be optimized so as to maximize the kurtosis of the wavelet coefficients in order to render the wavelet coefficients sensitive to the generated fault signals. An experimental data set which is representative of a localized gear tooth defect is used to compare the diagnostic capability of the fast Fourier transform power spectrum to the wavelet envelope power spectrum as respectively computed using Laplace and Morlet wavelet functions.

This paper is organized as follows: section 2 considers theory on the wavelet transform, section 3 explains the experimental setup and procedure, section 4 discusses the implementation of the enveloped wavelet power spectrum for gear fault detection and the conclusions are presented in section 5.

\section{Wavelet Transform}

\subsection{The Continuous Wavelet Transform:}

In wavelet analysis, a variety of different probing functions may be used, but the family always consists of enlarged or compressed versions of the basic function, as well as translations. Mathematically, the wavelet transform of a finite energy signal $\mathrm{x}$ $(t)$ with the analyzing wavelet $\psi$, leads to the definition of continuous wavelet transform as given in equation (1). 


$$
W(a, b)=\int_{-\infty}^{\infty} x(t) \frac{1}{\sqrt{|a|}} \psi *\left(\frac{t-b}{a}\right) d t
$$

Where $b$ (dilation parameter) acts to translate the function across $\mathrm{x}(\mathrm{t})$ and the variable $a$ (scaling parameter) acts to vary the time scale of the wavelet function $\psi$. The $*$ indicates the operation of complex conjugate and the normalizing parameter $1 / \sqrt{a}$ ensures that the energy is the same for all values of $a$. If $a$ is greater than one, the wavelet function $\psi$, is stretched and if it is less than one, it contracts the function along the time axis. Negative values of $a$ simply flip the probing function on the time axis (Semmlow, 2004). While the wavelet function $\psi$ could be any of a number of different functions, it always takes on an oscillatory form, hence the term "wavelet". The base wavelet is generated when $b=0$, and $a=1$, then the wavelet is in its natural form, which is termed the mother wavelet. The wavelet coefficients $W(a, b)$ are the similarity between the waveform and the wavelet at a given combination of scale and position $a, b$. Wavelet analysis can be thought of as a search over the waveform of interest for activity that most clearly approximates the shape of the wavelet. This search is carried out over a range of wavelet sizes: the time span of the wavelet varies although its shape remains the same. Since the net area of a wavelet is always zero by design, a waveform that is constant over the length of the wavelet would give rise to zero coefficients. Wavelet coefficients respond to changes in the waveform, more strongly to changes on the same scale as the wavelet, and most strongly, to changes that resemble the wavelet (Semmlow, 2004).

\subsection{Enveloped wavelet power spectrum:}

The envelope detection or amplitude demodulation is the technique of extracting the modulating signal from an amplitudemodulated signal. The result is the time-history of the modulating signal. Envelope analysis is the FFT frequency spectrum of the modulating signal. The vibration signal of a faulty gear can be viewed as a carrier signal at a resonant frequency of the gear modulated by a decaying envelope. The goal of the enveloping approach is to replace the oscillation caused by each impact with a single pulse over the entire period of the impact.

Laplace wavelet is a complex analytical and single sided damped exponential and is given in equation (2). The view of Laplace wavelet is shown in Figure 1

$$
\begin{array}{ll}
\psi(t)=\mathrm{A} \mathrm{e}^{-\left(\frac{\beta}{\sqrt{1-\beta^{2}}}+i\right) \omega_{c} t} \quad \mathrm{t} \geq 0 \\
\psi(t)=0 \quad \mathrm{t} \text { is other wise }
\end{array}
$$

Where $\beta$ is the damping factor that controls the decay rate of the exponential envelope in the time domain and hence regulates the resolution of the wavelet and it simultaneously corresponds to the frequency bandwidth of the wavelet in the frequency domain. The frequency $\omega_{c}$ determines the number of significant oscillations of the wavelet in the time domain and corresponds to wavelet center frequency in the frequency domain and A is an arbitrary scaling factor (Al-Raheem, 2007).

The optimal values of $\beta$ and $\omega_{c}$ for given vibration signal can be found by adjusting the time-frequency resolution of the Laplace wavelet to the decay rate and the frequency of the impulses to be extracted. Kurtosis is an indicator that reflects the impulsive content of a signal, and also it measures the divergence from a fundamental Gaussian distribution. A high kurtosis values indicates high impulsive content of the signal with more sharpness in the signal intensity distribution

Let $x(n)$ be a real discrete time random process, and $W T_{a}$ its $N$ point Laplace wavelet transform at scale a. The Laplace Wavelet Kurtosis (LWK) for $x(n)$ is defined as the kurtosis of the magnitude of $W T_{a}$ at each wavelet scale a as in the equation (3). (AlRaheem, 2011)

$$
L W K_{(a)}=\frac{\sum_{n=1}^{N} a b s\left(W T_{a}^{4}\left(x(n), \psi_{\beta}, \omega_{c}\right)\right)}{\left[\sum_{n=1}^{N} a b s\left(W T_{a}^{2}\left(x(n), \psi_{\beta}, \omega_{c}\right)\right)\right]^{2}}
$$

The Morlet wavelet is defined as given in equation (4) (Semmlow, 2004). The view of Morlet wavelet is shown in Figure 2

$$
\psi(t)=e^{-t^{2}} \cos \left(\pi \sqrt{\frac{2}{\ln 2}} \mathrm{t}\right)
$$

The wavelet transform (WT) of a finite energy signal $\mathrm{x}(\mathrm{t})$, with the mother wavelet $\psi(t)$, is the inner product of $\mathrm{x}(\mathrm{t})$ with a scaled and conjugate wavelet $\psi^{*}{ }_{a, b}$. Since the real and complex wavelet is employed to calculate the wavelet transform, the result of the wavelet transform is also an analytical signal as shown in equation (5) and (6).

$$
\begin{aligned}
& \mathrm{WT}\{x(t), a, b\}=<\mathrm{x}(\mathrm{t}), \psi_{\mathrm{a}, \mathrm{b}}(\mathrm{t})>=\frac{1}{\sqrt{a}} \int_{-\infty}^{\infty} x(t) \psi^{*}{ }_{\mathrm{a}, \mathrm{b}}(t) d t \\
& =\operatorname{Re}[\mathrm{WT}(a, b)]+i \operatorname{Im}[\mathrm{WT}(a, b)]=A(a, b) \mathrm{e}^{i \theta(a . b)}
\end{aligned}
$$


Where $\psi_{a, b}$ is a family of daughter wavelet, defined by the dilation parameter $a$ and the translation parameter $b$, the factor $1 / \sqrt{a}$ is used to ensure energy preservation. The time-varying function $A(a, b)$ is the instantaneous envelope of the resulting wavelet transform (EWT) which extracts the slow time variation of the signal, and is given by equation (7)

$$
\mathrm{A}(a, b)=\operatorname{EWT}(\mathrm{a}, \mathrm{b})=\sqrt{\{\operatorname{Re}[\mathrm{WT}(\mathrm{a}, \mathrm{b})]\}^{2}+\{\operatorname{Im}[\mathrm{WT}(\mathrm{a}, \mathrm{b})]\}^{2}}
$$

For each wavelet, the inner product results in a series of coefficients which indicate how close the signal is to that particular wavelet. To extract the frequency content of the enveloped correlation coefficients, the wavelet-scale power spectrum (SWPS) (energy/unit scale) is given by equation (8)

$$
\text { SWPS }(a, \omega)=\int_{-\infty}^{\infty}|S E W T(a, \omega)|^{2} d \omega
$$

Where SEWT $(a, \omega)$ is the Fourier transform of EWT $(a, b)$.

The total energy of the signal $x(t)$ is given in equation (9)

$$
\text { TWPS }=\int|x(t)|^{2} \mathrm{dt}=\frac{1}{2 \pi} \int_{-\infty}^{\infty} S W P S(a, \omega) d a
$$

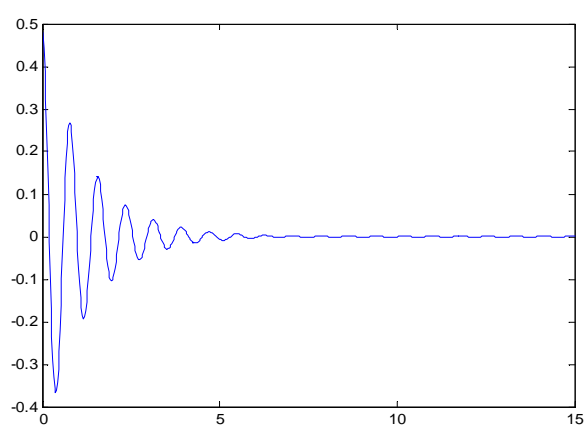

(a)

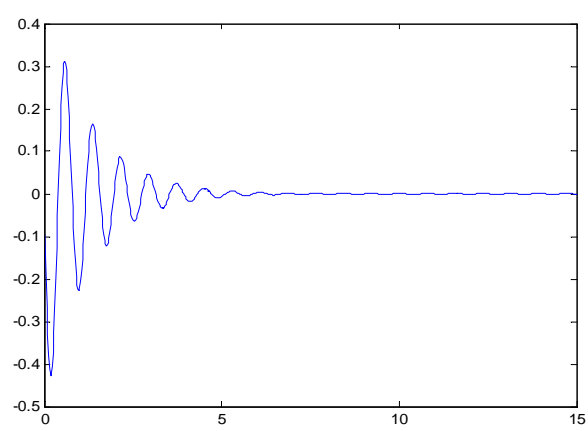

(b)

Figure 1. Laplace wavelet (a) Real part of Laplace wavelet (b) Imaginary part of Laplace wavelet

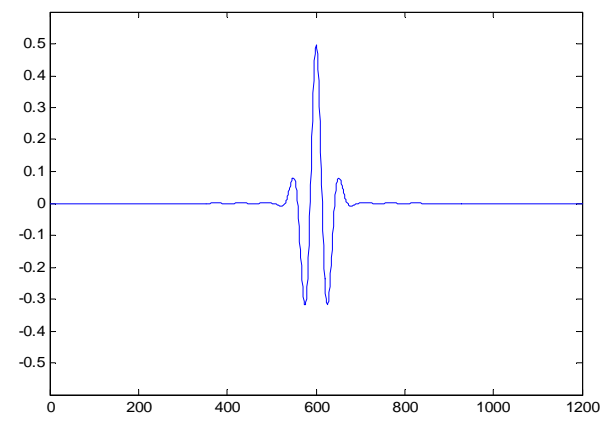

Figure 2. Morlet wavelet

\section{Experimental setup and procedure}

The fault simulator is depicted in Figure 3. The experimental setup consists of single stage gear box, motor, loading system, coupling and bearings. One gear was connected to 0.5 HP, 2900 RPM electric motor through coupling and the other gear was connected to a loading system. The gear and pinion has 46 and 23 teeth respectively. The shafts of $25 \mathrm{~mm}$ diameter connect gears with motor and loading system. The shafts are supported at its ends through bearings. The Gear mesh frequency (GMF) is calculated to be $23 * 2850 / 60=1092.5 \mathrm{~Hz}$. The vibration data is collected from the drive end bearing of gear box using the accelerometer (model 621B40, IMI sensors, sensitivity is $1.02 \mathrm{mV} / \mathrm{m} / \mathrm{s}^{2}$ and frequency range up to $18 \mathrm{kHz}$ ) with a NI Data Acquisition Device (NI-DAQ-National Instruments-NI SCXI-1000 chassis through SCXI-1530-channel 0, SCXI-1530-channel 1 and SCXI-1530-channel 2).The view of healthy gears is shown in Figure 4. The collected vibration data are exported as a data file to MATLAB software package for further processing. 


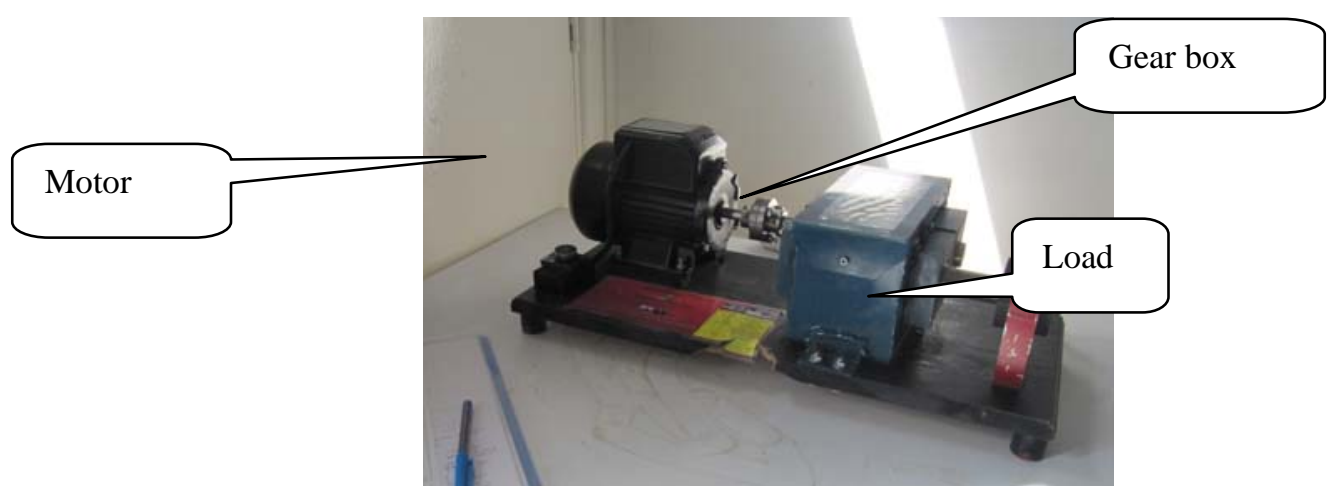

Figure 3. Fault Simulator set up.

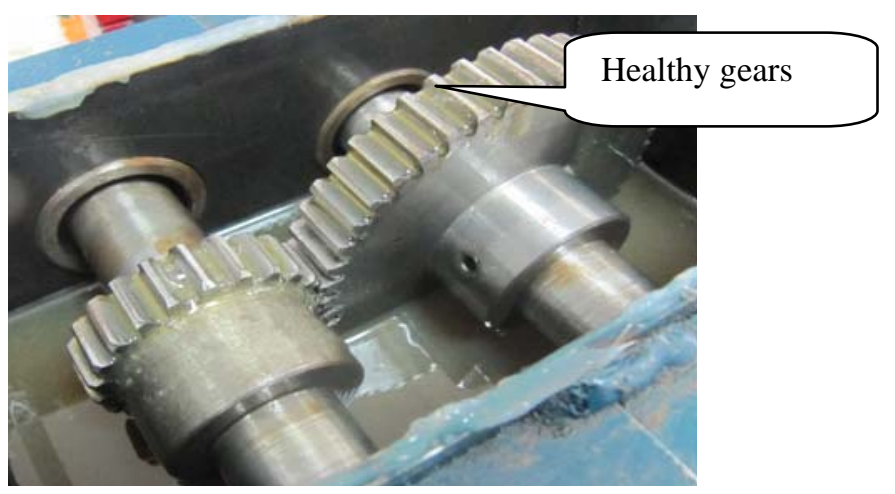

Figure 4.View of healthy gears

In the experimental investigation, the vibration signal was collected from a healthy gear at shaft speed of 2850 RPM under constant load condition. Further faults are induced in three different stages as shown in Table 1 and the corresponding vibration readings were taken. The views of faulty gear are shown in Figure 5. The sampling frequency of $16,000 \mathrm{~Hz}$ was used to collect the data for 2.4 seconds. The data was collected from the setup after reaching the required speed for different working conditions (lubrication, speed and load condition).

Table 1: Stages of induced fault

\begin{tabular}{|l|l|l|}
\hline Stage & Condition of the gear & Fault description \\
\hline Stage 0 & Healthy gear & Without any induced fault \\
\hline Stage 1 & Faulty gear & A crack of 3mm is induced at the root of the tooth \\
\hline Stage 2 & Faulty gear & Tooth was partially broken \\
\hline Stage 3 & Faulty gear & Tooth was completely removed \\
\hline
\end{tabular}

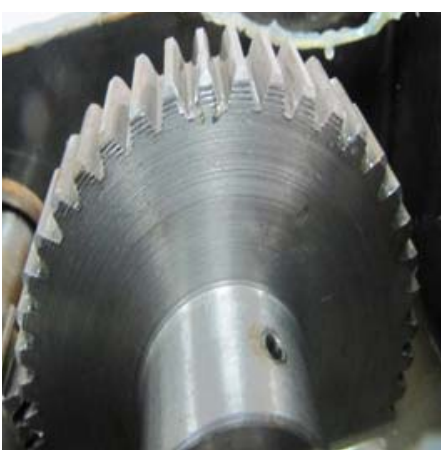

(a)

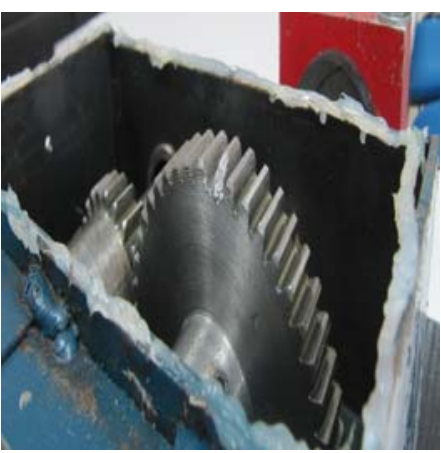

(b)

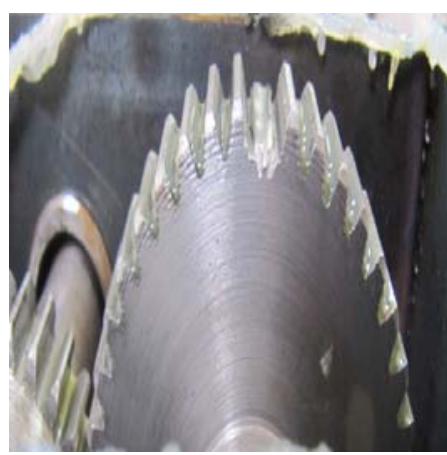

(c)

Figure 5. Gears with induced fault in 3 stages. (a) Stage 1. (b) Stage 2. (c) Stage 3 


\section{Implementation of wavelet power spectrum}

To visualize the performance of the proposed approach, this section presents several application examples for the detection of localized gear fault. It is well known that the most important components in gear vibration spectra are the tooth meshing frequency and its harmonics, together with sidebands due to modulation phenomena. The increment in the number and amplitude of sidebands indicate a fault condition. The methodology of implementing envelope power spectrum is shown in Figure 6. A typical time domain signal obtained from the experimental setup with gear fault, using accelerometer is given in Figure 7. This is further processed using various signal processing techniques based on FFT principle and enveloped power spectrum based on Morlet wavelet and Laplace wavelet. The GMF and its side bands are represented in various power spectrums with indication of data cursor value around $1093 \mathrm{~Hz}$, and $1047 \mathrm{~Hz}, 1140 \mathrm{~Hz}$ respectively. The rotational frequency of pinion, gear, GMF and side bands of GMF are depicted in Table 2.

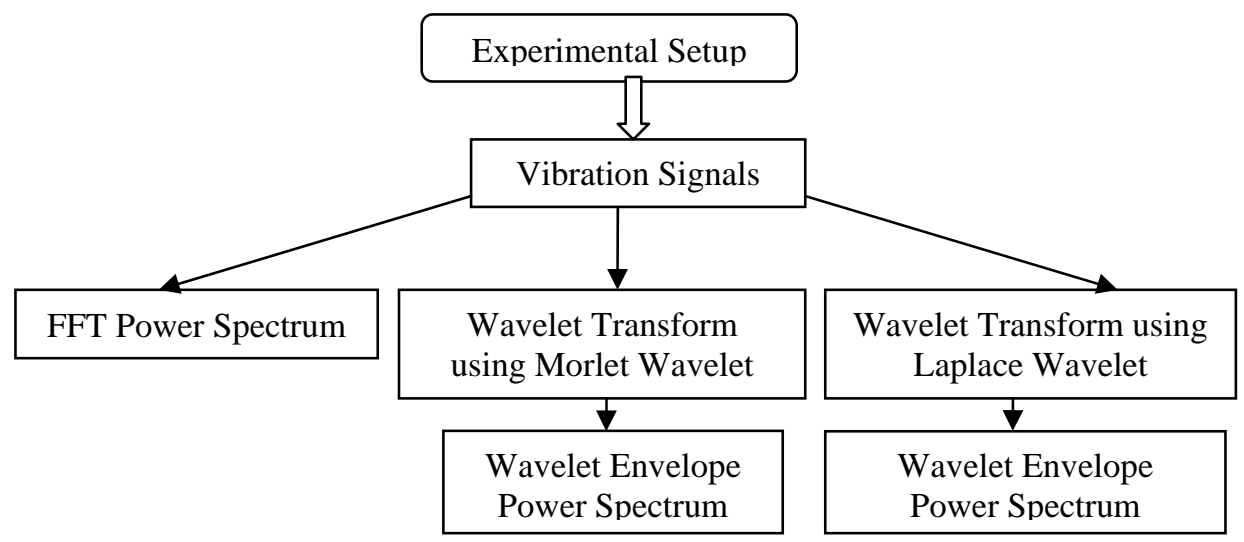

Figure 6. Methodology of implementing envelope power spectrum

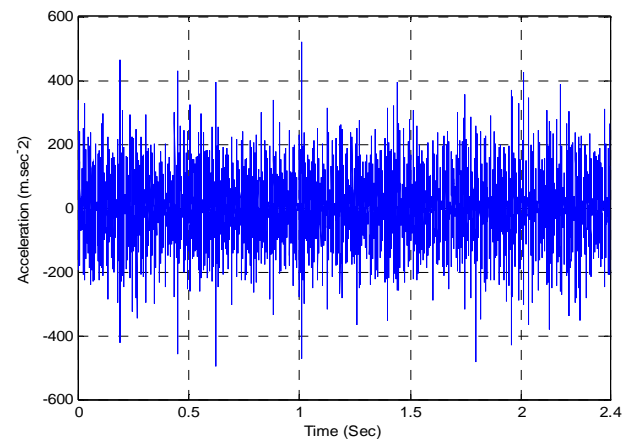

Figure 7. Time domain signal of gear with fault

Table 2. Frequency of rotation

\begin{tabular}{|l|l|}
\hline Frequency $(\mathrm{Hz})$ & Description \\
\hline 48 & Pinion rotational frequency \\
\hline 24 & Gear rotational frequency \\
\hline 1093 & GMF \\
\hline 1045 & Side band of GMF frequency \\
\hline 1140 & Side band of GMF frequency \\
\hline
\end{tabular}




\subsection{FFT Power Spectrum}

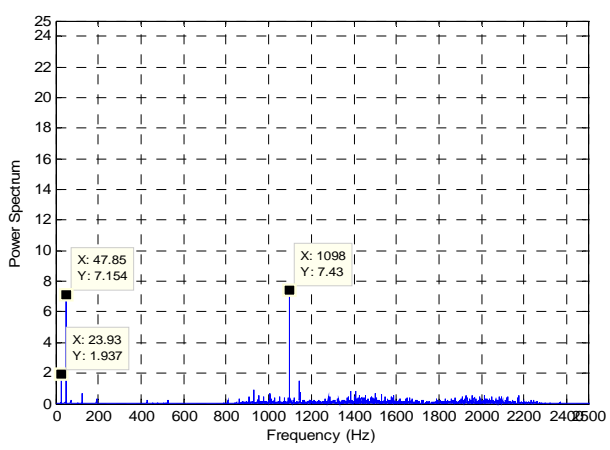

(a)

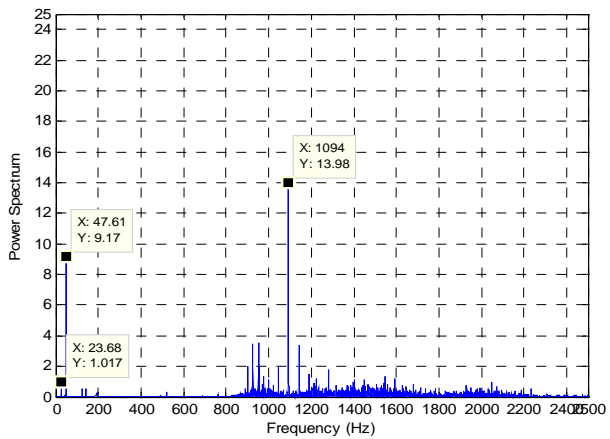

(b)

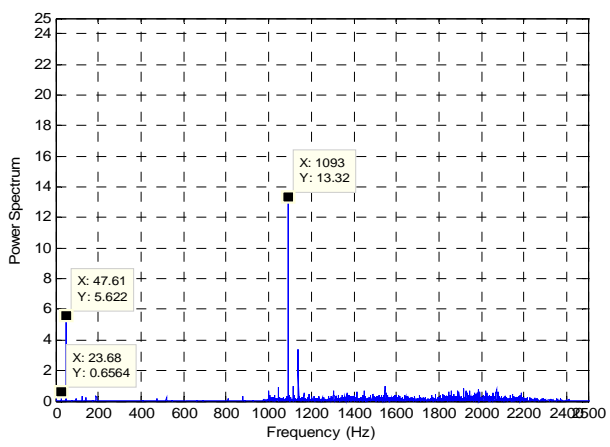

(c)

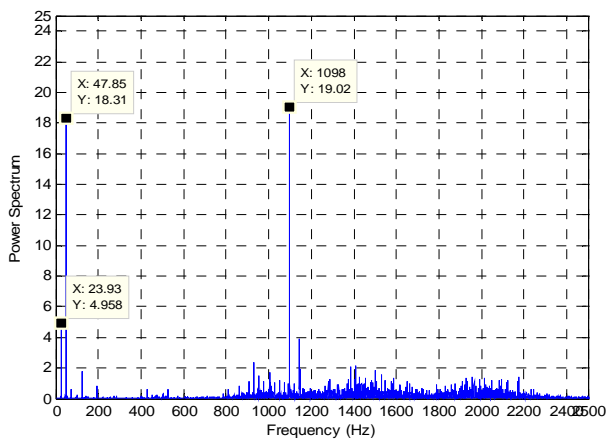

(d)

Figure 8. FFT Power Spectrums. (a) Without any defect. (b) $1^{\text {st }}$ stage of defect. (c) $2^{\text {nd }}$ stage of defect (d) $3^{\text {rd }}$ stage of defect

FFT power spectrums are shown for the healthy gear in Figure 8(a) and for three induced stages of faults in Figure 8(b)-8(d). The increase in the amplitude of GMF in line with the size of defect condition is seen in the power spectrum. However FFT power spectrum fails to give any clear indication of induced fault at sideband frequencies as the vibration amplitude at sidebands do not 
change significantly with the size of fault and are not noticeable, as this information is used as a tool to understand the severity of damage.

\subsection{Morlet Wavelet Based Enveloped Power Spectrum}

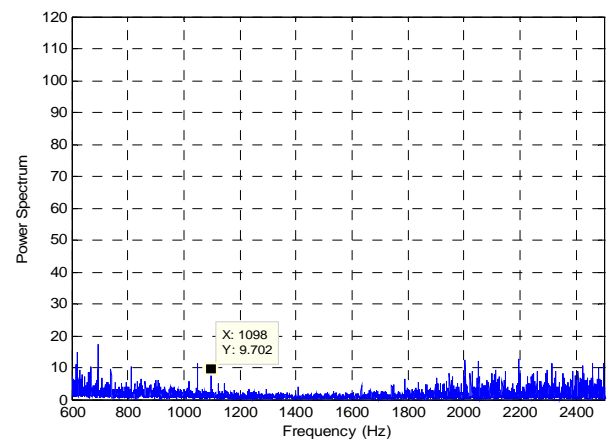

(a)

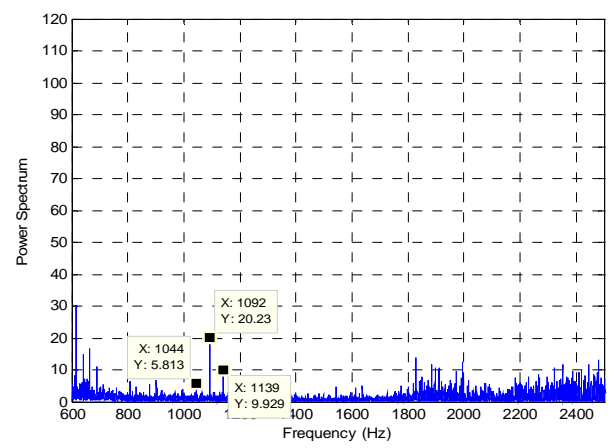

(b)

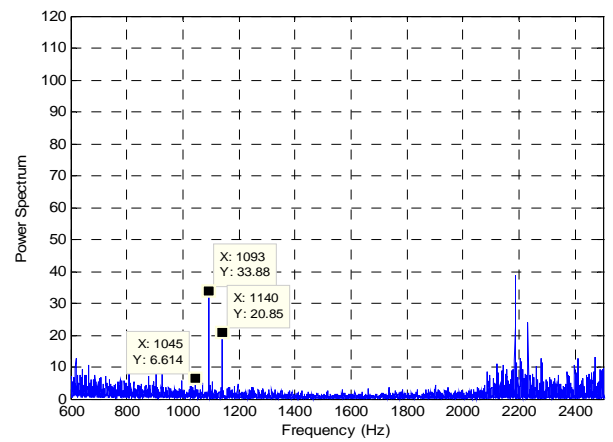

(c)

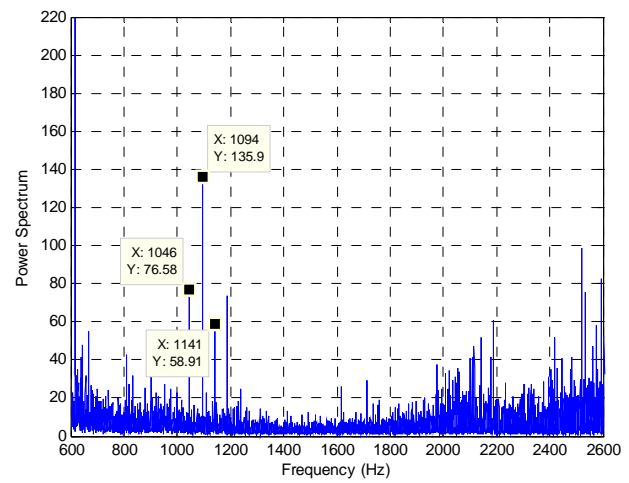

(d)

Figure 9 .Morlet Wavelet Based Enveloped Power Spectrums. (a) Without any defect. (b) $1^{\text {st }}$ stage of defect. (c) $2^{\text {nd }}$ stage of defect (d) $3^{\text {rd }}$ stage of defect 
Morlet wavelet enveloped power spectrums are shown for the healthy gear in Figure 9(a) and for 3 induced stages of faults in Figure 9(b) - 9(d). Amplitude of GMF is increasing from 9.7 to $135.9 \mathrm{~mm} / \mathrm{s}^{2}$ for healthy gear and faulty gear in 3 different stages. Vibration amplitude of side bands is significant. Changes in the number and strength of the side bands indicate severity of fault.

\subsection{Laplace Wavelet Based Enveloped Power Spectrum}

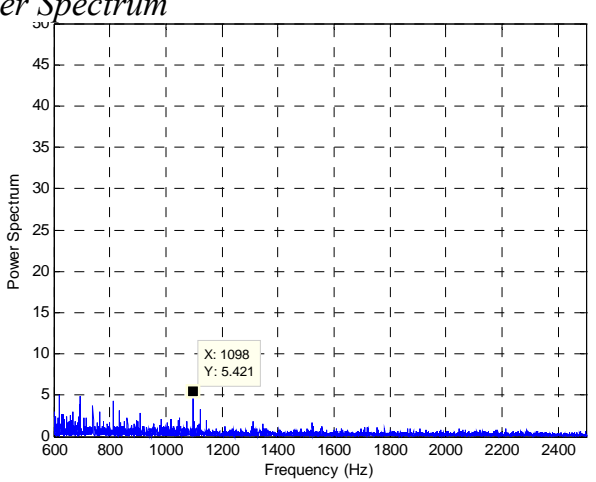

(a)

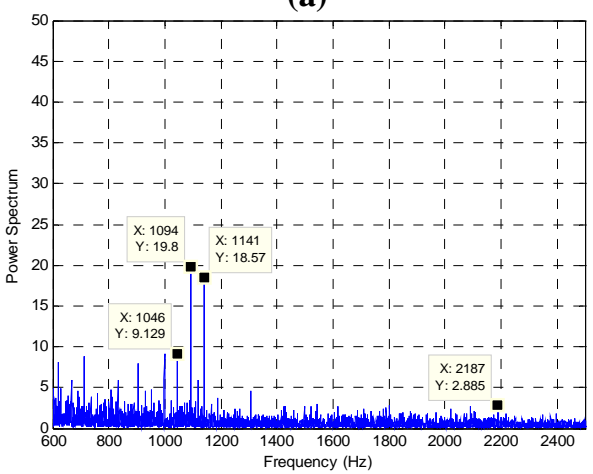

(b)

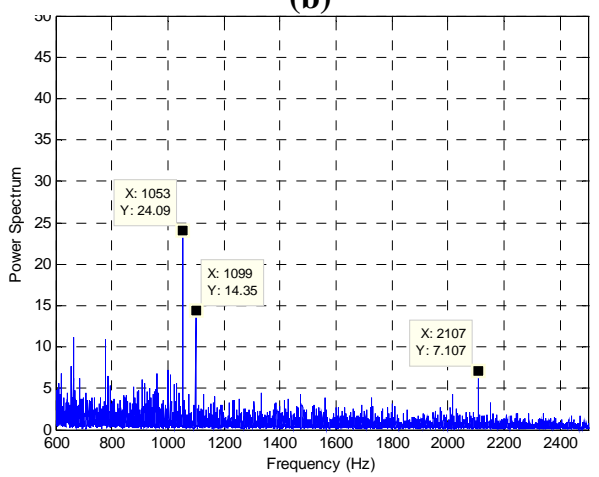

(c)

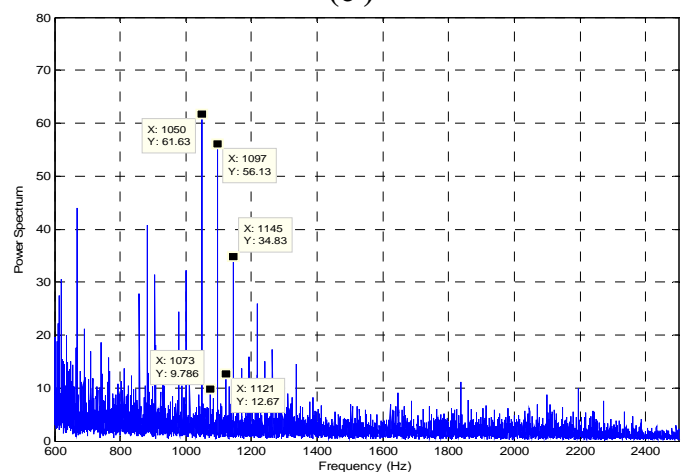

(d)

Figure 10. Laplace Wavelet Based Enveloped Power Spectrums. (a) Without any defect. (b) $1^{\text {st }}$ stage of defect. (c) $2^{\text {nd }}$ stage of defect (d) $3^{\text {rd }}$ stage of defect 
Laplace wavelet enveloped power spectrums are shown for the healthy gear in Figure 10 (a) and for faulty gear in three different stages of fault in Figure 10 (b)-10 (d).As seen in Laplace wavelet based enveloped power spectrums, vibration amplitude at GMF is increasing inline with the severity of fault from 5.4 to $56.1 \mathrm{~mm} / \mathrm{s}^{2}$ with dominant sidebands, indicating severity of fault. For a gear box in good condition, side band level remains low as seen in Figure 10(a). Laplace wavelet analysis is powerful in isolating peaks at sidebands of GMF, which can provide more precise information about defect condition.

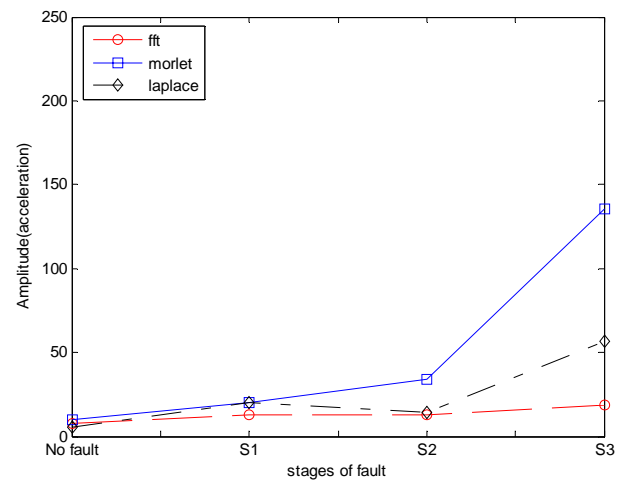

Figure 11. Vibration amplitude at GMF

Figure 11 shows the vibration amplitude peak trend at GMF for different stages of fault from various power spectrums. FFT power spectrum shows minimum increase in vibration amplitude peak at GMF and Morlet enveloped power spectrum shows considerable increase in comparison to other power spectrums. The proposed enveloped power spectrum technique is also applied for different lubrication, speed and loading conditions. The proposed technique has less influence for the different working conditions of the gear. The change in amplitude at GMF for different working conditions is shown in Table 2.

\begin{tabular}{|l|l|l|l|}
\hline Condition & FFT & Morlet wavelet & Laplace wavelet \\
\hline Full lubrication & 7.4 & 9.7 & 5.4 \\
\hline No lubrication & 6.41 & 32.2 & 11.8 \\
\hline Speed at 2665 RPM & 3.3 & 10.06 & 6.7 \\
\hline Speed at 2865 RPM & 7.4 & 9.7 & 5.4 \\
\hline With load & 3.7 & 48.3 & 23.4 \\
\hline Without load & 7.4 & 9.7 & 5.4 \\
\hline
\end{tabular}

\section{Conclusions}

The vibration signal processing techniques using FFT, Morlet wavelet enveloped power spectrum and Laplace wavelet enveloped power spectrum are implemented and their results are compared for various stages of induced gear fault. The proposed application shows the prominence of Morlet wavelet and Laplace wavelet based enveloped power spectrum for identification of fault in gear by comparing the significant increase in vibration amplitude at GMF and their 1xRPM of side bands compared to FFT power spectrum. The proposed diagnostic technique is less sensitive to changes in the operational speed, lubrication and load compared to standard FFT analysis. Implementing proper de-noising technique can further enhance the extraction of defect feature of gear.

\section{Acknowledgements}

The authors would like to thank management of Caledonian College Of Engineering, Sultanate of Oman for facilitating this work and their support. Also the authors would like to thank the reviewers for their comments and suggestions that helped in revising the paper to its present form.

\section{References}

Abu-Mahfouz I.A., 2007. Gearbox health monitoring experiment using vibration signals. Journal of Engineering Technology, Vol. 24, No. 1, pp. 26-31.

Al-Raheem K.F, Roy. A, Ramachandran.K.P. 2006. Detection of rolling element bearing faults using optimized -wavelet denoising technique. International Conference on Signal Processing, Beijing, China. 
Al-Raheem K.F., Roy A., Ramachandran K.P, Harrison D.K., Grainger S., 2009, International Journal of Advanced Manufacturing Technology, Vol. 40, pp. 393-402.

Al-Raheem K.F., Roy A., Ramachandran K.P, Harrison D.H., Grainger, S. 2007. Rolling element gearing fault diagnosis using Laplace-wavelet envelop power spectrum. EURASIP Journal on Advances in Signal Processing, Vol. 2007, article id 73629, 14pages

Al-Raheem K.F., Kareem W.A., 2011..Rolling element bearing fault diagnostics using Laplace wavelet kurtosis, IJMSE, World Academic Publishing, Vol.1 No.1, pp. 17-25.

Choy F.K., Huang, J.J., Zakrajsek R.F, Handschuh T.P, Townsend.1994. Vibration signature of a faulted gear transmission system. NASA, Technical Memorandum 106623, Technical Report ARL-TR-475 AIAA-94-2937.

Dalpiaz G., Rivola A. and Rubini R.,1998. Gear fault monitoring: comparison of vibration analysis techniques. Proceedings of the 3rd International Conference on Acoustical and Vibratory Surveillance Methods and Diagnostic Techniques, Vol. 13, pp, 623637.

Freudinger L.C., Lind R., and Brenner M.J., 1998. Correlation filtering of modal dynamics using the Laplace wavelet. Proceeding of the $16^{\text {th }}$ International Modal Analysis Conferences, Vol. 2, pp. 868-877.

Junsheng C., Dejie Y., Yu Y., 2005. Application of an impulse response wavelet to fault diagnosis of rolling bearings, Mechanical Systems and Signal Processing, Vol. 21, No. 2, pp. 920-929.

Lin J, Zuo M.J., 2003. Gear box fault diagnosis using adaptive wavelet filter, Mechanical Systems And Signal Processing, Vol. 17, No. 6, pp. 1259-1269.

Nikolaou N.G and Antoniadis I.A., 2002. Demodulation of vibration signals generated by defects in rolling element bearings using complex shifted Morlet wavelets. Mechanical Systems and Signal Processing, Vol. 16, No. 4, pp. 677-694.

Omar F.K. and Gaouda A.M.,2009. Gearbox diagnostics using wavelet based windowing technique. Journal of Physics: Conference Series, Vol. 181, No. No. 1, Conf. Ser. 181012089

Peng Z.K., Chu F.L., 2004. Application of the wavelet transform in machine condition monitoring and fault diagnostics: a review with bibliography. Mechanical Systems and Signal Processing, Vol. 18, No. 2, pp. 199-221.

Prokop M., Mohr F. 2003. Gear box vibration analysis, University of Applied Sciences Pforzheim, Germany online.

Pan M.C., Sas P., 1996. Tansient analysis on machinery condition monitoring. International Conference on Signal Processing, Proceedings, ICSP 2, pp. 1723-1726.

Purushotham V., Narayanan S, and Suryanarayana, Prasad A.N., 2005 Multi-fault diagnosis of rolling bearing elements using wavelet analysis and hidden Markov model based fault recognition. NDT \& E International Vol. 38, No. 8, pp. 654-664.

Randall R.B. 1982. A new method of modeling gear faults. Journal of Mechanical Design, Vol. 104, pp. 259-267.

Saravanan N, Kumar V.N.S., Siddabattuni, Ramachandran K.I., 2010. Fault diagnosis of spur bevel gear box using artificial neural network and proximal support vector machine, International Journal of Applied Soft Computing, Vol. 10, pp. 344-360.

Saravanan N. Ramachandran K.I.,.2010. Incipient gear box fault diagnosis using discrete wavelet transform for feature extraction and classification using artificial neural network. An international journal of Expert Systems with Applications, Vol. 37, pp. 4168-4181.

Semmlow J.L. 2004. Bio signal and biomedical image processing, Marcel Dekker, Inc. New York, USA, ISBN0-8247-4803-4

Shi D.F., Wang W.J. and Qu L.S., 2004. Defect detection for bearing using envelope spectra of wavelet transform. ASME $J$ of Vibration and Acoustics, Vol. 120, pp. 567-574.

Smith C., Akujuobi C.M., Hamory P., Kloesel K., 2006. An approach to vibration analysis using wavelets in an application of aircraft health monitoring, Mechanical Systems and Signal Processing, Vol. 21, pp. 1255-1272.

Tse P.W., Wen-Xian Yang, Tam.H.Y, 2004. Machine fault diagnosis through an effective exact wavelet analysis. Journal of Sound and Vibration, Vol. 277, pp.1005-1024.

van Khang N., Cau T.M., Dien N.P., 2004. Modeling parametric vibration of gear pair systems for a tool for aiding gear fault diagnosis, Technische Mechanic, Band 24, Heft 3-4, pp. 198-205.

Wandel J.. 2006. Multistage gearboxes: Vibration based quality control, KTH Engineering Sciences, Stockholm, Licentiate Thesis, TRITA-AVE 2006:27 ISSN 1651-7660.

Yanyang Z., Xuefeng C. Zhengila H. and Peng C., 2005. Vibration based modal parameters identification and wear fault diagnostics using Laplace wavelet. Key Engineering Materials, Vol. 293-294, pp. 183-190.

\section{Biographical notes}

M.Lokesha received his B.E. degree in Mechanical Engineering from Mysore University, Mysore, India in 1989 and M. Tech. degree in maintenance engineering from Mysore University, Mysore, India in 1994. Presently, he is working as a Senior Lecturer in the Department of Mechanical and Industrial Engineering at Caledonian College of Engineering, Muscat. He has been working for 20 years in engineering institutions. His research focuses on vibration instrumentation \& measurement as well as condition monitoring of rotating machineries.

Dr Manik Chandra Majumder is a Professor in the Department of Mechanical Engineering and Member Secretary of the Senate, National Institute of Technology, Durgapur, India. He has a PhD from the Indian Institute of Technology, Kharagpur, India. He has guided many Ph.D. scholars. His prime area of research is Tribology. 
Dr. K. P. Ramachandran B.E, M.Tech, PhD is currently working as Associate Dean (Post Graduate Studies \& Research), Caledonian College of Engineering, Muscat, Sultanate of Oman. He has been working for more than 25 years in engineering institutions and as a consultant for many industries. He has research interest in the vibration instrumentation \& measurement, analysis and control, condition monitoring of rotating machinery. He has many publications to his credit and has been conferred Sir C.V. Raman award for the best technical paper published in the Journal of Vibration \& Acoustics (1997). He is on the editorial board and technical reviewer for many international journals and conferences. He has guided many PhD students in the area of condition monitoring and maintenance management.

Dr.Khalid Fatihi Abdul-Raheem, B.E., M.Tech from University of Technology, Iraq and PhD from Glasgow Caledonian University, UK. Presently he is working as an Associate Professor in the Department of Mechanical and Industrial Engineering at Caledonian College of Engineering, Muscat. He has been working for more than 22 years in engineering institutions and many industries. He has many publications to his credit. He has research interest in condition monitoring and signal analysis, artificial intelligence, automatic control system, mechanical vibration analysis and control.

Received December 2011

Accepted March 2012

Final acceptance in revised form May 2012 